\title{
Effect of $\beta$-Cyclodextrin Stabilized Silver Nanoparticles on Gills, Kidney, Liver of Danio rerio
}

\author{
Bipin Lade 1(D), Arti Shanware 1, Ritika Sharma ${ }^{1, *}$ \\ 1 Rajiv Gandhi Biotechnology Centre Rashtrasant Tukadoji Maharaj Nagpur University, LIT Campus, Nagpur 440033, \\ Maharashtra, India \\ * Correspondence: dbipinlade@gmail.com (B.L.);
}

Scopus Author ID 56287462400

Received: 15.04.2021; Revised: 5.06.201; Accepted: 8.06.2021; Published: 27.06.2021

\begin{abstract}
The silver nanoparticles (AgNP) are applied broadly in medical applications due to their antimicrobial property. However, the toxicity (uptake, translocation, and accumulation) of these AgNPs nanomaterial has not been much explored. Also, cyclodextrin has been used for different pharmaceutical applications due to its various potential properties. Therefore, the toxicity of these AgNPs and cyclodextrin in the model organism such as Danio rerio could be crucial for future nanodrug studies. The main aim of the present research work is to synthesize, characterize biopolymeric silver nanoparticles, and perform histopathological studies of synthesized silver nanoparticles on Danio rerio. The silver nanoparticles of $130 \mathrm{~nm}$ size have been synthesized successfully using $\beta$-Cyclodextrin $(\beta-C D)$ at room temperature. The various analytical applied to characterized the $\beta$-Cyclodextrin $(\beta-C D)$ reduced silver nanoparticles ( $\beta$-CD)-SNPs. A histopathological study has been conducted to evaluate the toxicity effect of the synthesized ( $\beta$-CD)-SNP on the animal model Danio rerio. The ( $\beta$-CD)-SNPs conc. 30 $\mu 1 / 1$ is affecting and damaged gills and kidney the Danio rerio organs (gills, kidney) exposed after 10 days, yet the liver was found to be healthy. In conclusion, the Danio rerio gills, kidneys, and liver are sensitive to the $130 \mathrm{~nm}$-sized $(\beta-\mathrm{CD})$-SNPs. The nanoparticles' toxicity depends on concentration; less concentration $(30 \mu \mathrm{l} / \mathrm{l})$ accumulates and is absorbed efficiently than the higher concentration $(300 \mu 1 / 1)$. However, no morphological changes were observed on Danio rerio.
\end{abstract}

Keywords: silver nanoparticles; $\beta$-Cyclodextrin ( $\beta-\mathrm{CD})$; Zebrafish; histopathological study.

(C) 2021 by the authors. This article is an open-access article distributed under the terms and conditions of the Creative Commons Attribution (CC BY) license (https://creativecommons.org/licenses/by/4.0/).

\section{Introduction}

Nanoparticles are defined as particles with a diameter between 1 and $100 \mathrm{~nm}$ or fibers spanning the range 1-100 $\mathrm{nm}$ as per given by the International Organization for Standardization, American Society of Testing and Materials, and National Institute of Occupational Safety and Health. A bottom-up synthesis method implies that the nanostructures are synthesized onto the substrate by stacking atoms onto each other, which gives rise to crystal planes, crystal planes further stack onto each other, resulting in the synthesis of the nanostructures $[1,2]$. Nanoparticles are also fueling a new discipline of nanomedicine, lying at the intersection of chemistry, physics, and medicine and focused on a range of biomedical applications [3]. Nanoparticles are also used in various therapeutic applications, bioimaging, drug delivery, tissue engineering, and widespread importance in research [4, 5]. Some of the types of nanoparticles are carbon-based nanoparticles, ceramic nanoparticles, metal nanoparticles, semiconductor nanoparticles, polymeric nanoparticles, and lipid-based 
nanoparticles. These metallic nanoparticles (gold, silver, aluminum, copper, iron, zinc) are important and used largely for research and application.

\subsection{Silver nanoparticles (SNPS).}

Silver nanoparticles nowadays are filling the role of silver sulfadiazine as an advanced wound treatment agent. They are typically created by reducing a silver salt with a reducing agent such as sodium borohydride in the presence of a colloidal stabilizer. Polyvinyl alcohol, poly (vinyl pyrrolidone), bovine serum albumin (BSA), citrate, and cellulose are the most commonly used colloidal stabilizers [6]. The silver nanoparticles (SNPs) belong amongst the most frequently used nanoparticles due to their antimicrobial, anticancer, larvicidal, catalytic, and wound healing activities [7]. Large numbers of papers use polymers such as chitosan, polylactic-co-glycolic acid, cationic polymers, cellulose, agar, gum, gelatin, pectin, pullulan, sodium alginate, zein, cyclodextrin, and plant-based material for silver nanoparticles synthesis etc. $[2,4-8]$. Among these polymers and polysaccharides, cyclodextrin has played an important role in medicine and pharmaceutical.

\subsection{Cyclodextrin.}

Cyclodextrins are cyclic oligosaccharides composed of $(-1,4)$ connected -Dglucopyranose units with a lipophilic central cavity and a hydrophilic outer surface. The cyclodextrins are shaped like truncated cones rather than a complete cylinder due to the chair conformation of the glucopyranose units [8]. The primary hydroxyl groups of the sugar residues are found outside the cone, with secondary hydroxyl groups at the broad edge. The center cavity is lipophilic because it is walled by the glucose residues' skeletal carbons and ethereal oxygens. The cavity has been predicted to have a polarity similar to that of an aqueous ethanolic solution. They are stable in an alkaline medium, hydrolyze in a strongly acid medium, and are resistant to enzymatic degradation by $\beta$-amylase. The potential of cyclodextrin to change the physicochemical and biological properties of drugs is its most important characteristic. This cavity can establish interactions through intermolecular forces with molecules, ions, or radicals, acting as a host substance and the resulting molecular complex is called an inclusion compound or a supramolecular compound [9]. There are three types of natural cyclodextrin which are $\alpha$-, $\beta$ - and $\gamma$-cyclodextrin consist of six, seven, and eight glucopyranose units, respectively.

\subsection{Beta-cyclodextrins $(\beta-C D)$.}

Beta-cyclodextrin ( $\beta-C D)$ is a cyclodextrin composed of seven alpha-(1->4) linked Dglucopyranose units. The cyclodextrins are a group of structurally related natural products formed during the bacterial digestion of cellulose. Its molecular formula is $\left(\mathrm{C}_{6} \mathrm{H}_{10} \mathrm{O}_{5}\right)_{7}$; the molecular weight is $1135 \mathrm{~g} / \mathrm{mol}$, and solubility $18.5 \mathrm{mg} / \mathrm{l}$. Figure 1 shows the structure of the $\beta$-cyclodextrin, which was obtained from PubChem CID: 444041.
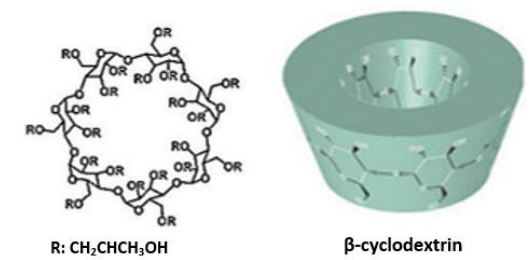

Figure 1. Molecular and 2D structure of $\beta$-Cyclodextrin. 
$(\beta-C D)$ has the lowest solubility due to the high number of intramolecular hydrogen bonds among secondary hydroxyl groups inside the molecule. These interactions make the structure rigid and prevent hydration by water molecules $[8,10]$. The $\beta$-cyclodextrins are able to form inclusion complexes with many drugs lipophilic moiety of the molecule in the central cavity without formation and destruction of covalent bonds, and the drug molecules in the complex are in rapid equilibrium with free molecules in the solution. The driving forces for the complex formation include the release of enthalpy-rich water molecules from the cavity, electrostatic interactions, Van der Waals interactions, hydrophobic interactions, hydrogen bonding, release [9].

The $\beta$-cyclodextrin reduced silver nanoparticles may be effective for applications in pharmaceutical industries for antibacterial, antifungal, enhanced activity, improved solubility, slow release of silver nanoparticles for long-term effects. However, their actual toxicity in humans is needed to be studied. Therefore, the model organism is the best way to see the optimistic as well as the negative effects. Danio rerio, a tropical freshwater fish famously known as zebrafish, is an indispensable animal model for scientific studies.

\subsection{Zebrafish (Danio rerio).}

Danio rerio, commonly known as zebrafish, is a tropical freshwater fish and the name zebrafish comes from the blue stripes that run horizontally on each side of their bodies. The first report of Danios in the literature comes from the surveys of South Asian flora and fauna by the Scottish physician and naturalist Francis Hamilton, where he noted that they were "beautiful fish" and "insipid" and likely of little economic value. They are commonly found in the tropics, although they are native to South Asia (Nepal, India, etc.). The study of zebrafish as a leading model organism for developmental biology is rapidly expanding. Several key features have led to their rapid growth as a research model, including their ease of care, fecundity, rapid development, small size, and ease of manipulation. The wide sharing of techniques, reagents and fish lines within the zebrafish research community has facilitated the rapid growth in research using zebrafish, with a dramatic rise in publications using zebrafish in recent years [11]. Researchers are trying to understand the genetic and neuronal basis for behavior in zebrafish. As such, they are particularly important in developmental biology, toxicity, biomedicine, and neurophysiology studies as model organisms [12].

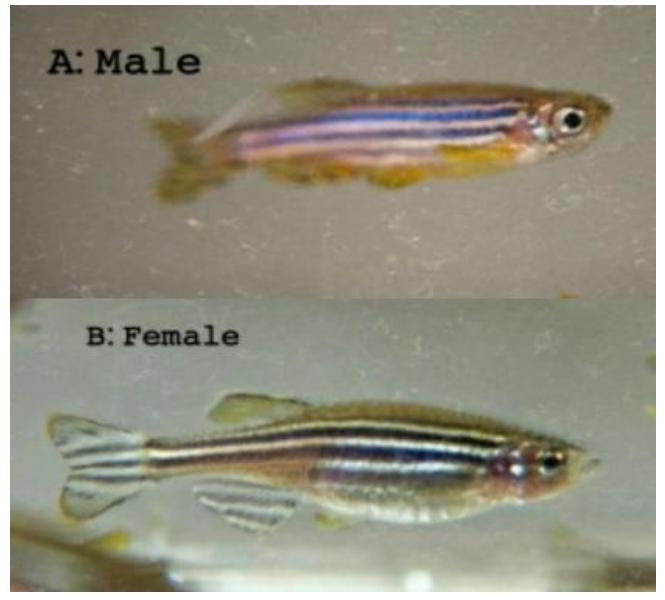

Figure 2. Adult (A: male, B: female) experimental zebrafish used in the present study and the fish tanks set-up. A: male AB strain, with classical striping, short fins and sleeker belly, B: female AB strain, with classical striping, short fins and rounder belly demonstrating that she is gravid. 
Apart from the fact that zebrafish share much of their genome with human beings, some of the other facts that make them ideal for research studies in genetic manipulation include laying many eggs, which is ideal for research studies. They can be easily bred in the laboratory. In addition, about 70 percent of diseases related to human genes can be found in zebrafish [13]. There are a lot of papers that present nanoparticle synthesis using polymer and their effects on zebrafish. However, we did not find any reports on the study of nanoparticles that are synthesized using beta-cyclodextrin toxicity effects on zebrafish. Thus, this present work was performed to synthesize and characterize the beta-cyclodextrin (important pharmaceutical polymer) reduced silver nanoparticles and study their histopathological effects on various organs of zebrafish.

\section{Materials and methods}

The materials, reagents, chemicals, glassware and equipment that used in the present study are silver nitrate $\left(\mathrm{AgNO}_{3}\right), \beta$-Cyclodextrin, haematoxylin-Eosin (HE), acetone, acetonitrile, $100 \%$ alcohol, distilled water, tween 20, dimethyl sulfoxide (DMSO), N-N dimethylformamide, sodium hydroxide $(\mathrm{NaOH})$, tricaine methanesulfonate, PBS Buffer, xylene and paraffin wax.

\subsection{Zebrafish: tank, condition, diet, and maintenance.}

Animal: Zebrafish were purchased from the A. K. Aquarium \& Pets shop located Model Mill Square, Ganeshpeth, Nagpur, Maharashtra 440018, India. Fish Tanks: Fish tanks set-up (Figure 2) involved 4 aquariums (three exposed and one control tank), each of 40 L capacity measuring $2 \times 30 \times 30 \mathrm{~cm}$ (length, width, and height). Zebrafish wild-type (Danio rerio) adults (6-8 months, 3-4 g) were obtained from a local commercial supplier and acclimated for at least 2 weeks in the experimental room before the experiments. Animals were housed in a $10 \mathrm{~L}-$ thermostated aquarium filled with unchlorinated water constantly aerated at a targeted temperature of $26 \pm 2{ }^{\circ} \mathrm{C}$ [14]. For between 4-5 fish, a $40 \mathrm{~L}$ tank was used for sufficient space for the fish to be social and to avoid aggressiveness. The same number of males to that of the female was taken care of, with each aquarium having 5 fishes, 3 males, and 2 females.

\subsubsection{Dark and light conditions.}

Fish were kept under a 14-h light/10-h dark cycle photoperiod (lights on at 10:00 am) and were fed twice a day with a commercial flake fish food supplement. The housing tank mimicked the conditions mentioned above and had a glass partition that allowed manipulated and non-manipulated fish to be maintained separated during each experimental session and yet allowed animals to be maintained among their original group during the investigation. This strategy was adopted to minimize animal stress due to isolation and its eventual impacts on behavioral responses.

\subsubsection{Feeding and oxygen supply.}

The feeding was not interrupted during the experimentation, and all sessions were performed in the morning. On the alternate session, animals were gently captured from the temporary housing tank using a $6 \mathrm{~cm}$ wide fine nylon mesh fish net. The oxygen was supplied through pumps installed inside the four aquariums. Water used in the experiments was obtained from a reverse osmosis apparatus. 


\subsubsection{Maintenance.}

During fish maintenance, water parameters were monitored daily and maintained in the following ranges: $\mathrm{pH}$ : $6.5-7.5$ and temperature: 25 to $28{ }^{\circ} \mathrm{C}$ with constant daily nutrition and natural physical/chemical conditions as photoperiod ensuring the highest animal welfare as it established by the World Organization for Animal Health. Ideal conditions were also maintained by supplying mechanical filters that remove large debris, activated carbon filters that remove contaminants, and small elements and biological filters that remove organic wastes.

\subsection{Synthesis of $\mathrm{AgNO}_{3}$.}

The $1 \mathrm{Mm} \mathrm{AgNO}_{3}$ solution is prepared, for which $1 \mathrm{ml}$ of prepared $100 \mathrm{ml}$ of $0.1 \mathrm{M}$ $\mathrm{AgNO}_{3}$ solution is taken and added in $99 \mathrm{ml}$ autoclaved distilled water. The lights were switched off for the preparation of $\mathrm{AgNO}_{3}$ solution because if silver nitrate is exposed to light falls, its color changes to brown or black. Dark (Brown color) bottles prevent the passage of light, and therefore it prevents the decomposition process.

\subsubsection{Synthesis of silver nanoparticles using $\beta$-cyclodextrin $(\beta-C D)$.}

For the synthesis of $\beta$-cyclodextrin silver nanoparticles ( $\beta$-CD)-SNPs, the $5 \mathrm{ml}$ of $1 \% \beta$ cyclodextrin solution was added to $95 \mathrm{ml}$ of aqueous silver nitrate solution $(1 \mathrm{mM})$. The $\beta$ cyclodextrin acts as a stabilizing agent in the solution and leads to the formation of ( $\beta-C D)$ SNPs. After few minutes, $1 \mathrm{~N} \mathrm{NaOH}$ solution was added to the mixture solution to maintain alkaline $\mathrm{pH}$ (Figure 3).
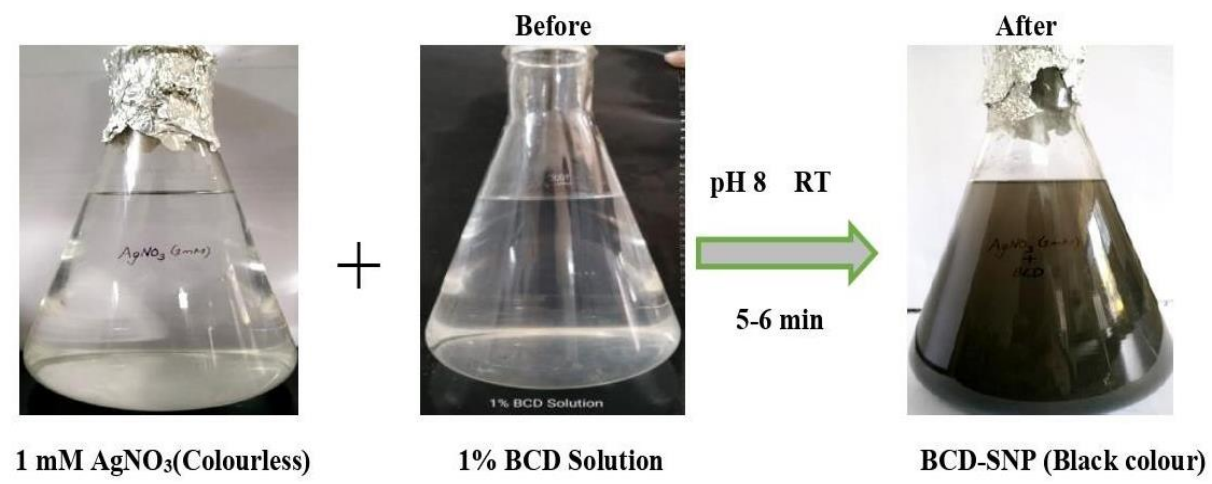

Figure 3. Step wise process for the preparation of $\beta$-cyclodextrin reduced silver nanoparticles ( $\beta$-CD)-SNPs.

\subsection{Characterization of $(\beta-C D)-S N P$.}

\subsubsection{Ultraviolet-Visible (UV-Vis) spectroscopy analysis.}

The $1 \mathrm{~mL}$ of the sample of the suspension was collected systematically to monitor the completion of bio-reduction of silver ions in aqueous solution, followed by dilution of the samples with $2 \mathrm{ml}$ of deionized water and subsequent scan in UV- Vis spectra, between wavelengths of 300 to $800 \mathrm{~nm}$ in the spectrometer and the UV-Visible spectra of the $(\beta-C D)$ SNPs solution was recorded.

\subsubsection{Fourier Transform Infra-Red Spectroscopy (FTIR).}

The nanoparticles were analyzed by attenuated total reflectance FTIR using HappGenzel at an infrared range of $650-4000 \mathrm{~cm}^{-1}$. The analysis of dried ( $\left.\beta-\mathrm{CD}\right)$-SNPs carried out 
through the potassium bromide $(\mathrm{KBr})$ pellets, FTIR grade method in 1:100 ratio and spectrum were recorded using a Jasco FT/IR-6300 Fourier transform infrared spectrometer equipped with JASCO IRT-7000 Intron Infrared Microscope resolution of $4 \mathrm{~cm}^{-1}$ transmittance (JASCO, Tokyo, Japan).

\subsubsection{Nanoparticle Tracking Analysis (NTA).}

The size of synthesized nanoparticles was measured by Nanosight (LM20, UK), where the $0.5 \mathrm{ml}$ of samples is diluted with the nuclease-free water and injected onto the sample chamber and observed through nanoparticles tracking to measure the size and distribution of the nanoparticles as point-scattering moving under Brownian motion.

\subsubsection{Zeta-potential analysis.}

The surface zeta potential was measured using the laser zeta meter (Malvern zeta seizer 2000, Malvern) were, samples of the nanoparticles $(5 \mathrm{ml})$ were diluted with double distilled water $(50 \mathrm{ml})$ using $\mathrm{NaCl}$ as a suspending electrolyte solution to adjust the $\mathrm{pH}$ to the required value. The sample was shaken for $30 \mathrm{~min}$. After shaking, the zeta potential of the metallic particles was measured.

\subsubsection{Exposure of zebrafish to ( $\beta$-CD)-SNPs.}

Adult animals, aged between 6 and 7 months, were exposed to silver nanoparticles with nominal concentrations of 0 (control group), 30,150, and $300 \mu \mathrm{l} / \mathrm{L}$ in an aquarium filled with 10L unchlorinated water ( 5 animals per tank) for a period of 30 days (solutions were not changed during this period).

\subsubsection{Experimental phase.}

Zebrafish were maintained in tanks equipped with aeration and fed daily. The fish behavior was evaluated visually after $0,3,6$, and $24 \mathrm{~h}$ and following 1-2 weeks after exposure to silver nanoparticles in order to reveal a change in swimming speed, loss of balance, and abnormal behavior.

\subsubsection{Light microscope.}

Light, or optical, microscopes were used for histological studies that rely on glass lenses and visible light to magnify tissue samples. The specimen preparation is performed with a fixation procedure to prevent degradation of the sample by naturally occurring enzymes that are released by the cells upon death. Once fixed, samples are placed into an embedding in paraffin wax medium to support the sample. However, glycerin-based freezing medium and agar were used to surround the sample during sectioning using microtome to shave the sample into thin slices ranging from a few microns to a few millimeters in thickness. Further, they are mounted on a glass slide and stained to bring out specific sample features before being imaged on a microscope.

\subsubsection{Histopathology.}

The adult zebrafish has been carefully euthanized by anesthesia with a dose of tricaine methanesulfonate (MS-222) buffer. Gills, liver, kidney were excised and immediately fixed in 
$4 \%$ formaldehyde in PBS buffer (Phosphate Buffer Saline) at room temperature for $24 \mathrm{~h}$. Gills were decalcified, prior to processing, with a decalcifier agent for $2 \mathrm{~h}$ at room temperature. Histological examinations were performed according to standard laboratory procedures. All tissues were washed three times with PBS $(0.1 \mathrm{M}, \mathrm{pH} 7.4)$, gradually dehydrated in ascending alcohols series $(35 \%, 50 \%, 70 \%, 95 \%$, absolute ethanol) for $20 \mathrm{~min}$ each one and clarified in xylene for $1 \mathrm{~h}$ at room temperature, subsequently embedded in paraffin wax (Medite tissue wax $56-58^{\circ} \mathrm{C}$ ). Five $\mu \mathrm{m}$ thick histological sections were cut by microtome (Reichert Jung 1150 Autocut) and collected on glass slides (Menzel Glaser, Thermo Scientific). At least 2 slides of each tissue were collected. The sections were stained with Haematoxylin-Eosin (HE) (BioOptica) and observed under an optical microscope to identify potential morphological alterations, and the photographs were then taken with a mobile camera (Redmi Note 6 Pro) [15].

\section{Results and Discussion}

\subsection{Preliminary confirmation of $(\beta-C D)-S N P$ s.}

The synthesis of ( $\beta$-CD)-SNPs was confirmed by visual observation in which silver nitrate solution $(1 \mathrm{mM})$ was colorless, which changed from dark grey color to black color after the reaction.

\subsection{Characterization of ( $\beta$-CD)-SNPs.}

The synthesis of $(\beta-C D)-S N P s$ was characterized using Shimadzu 1800 UV-Vis spectrophotometer, Fourier-transform infrared (FTIR) spectroscopy, zeta potential, and nanoparticle tracking analysis (NTA).

\subsubsection{UV-Vis Spectroscopy analysis of ( $\beta$-CD)-SNPs.}

The synthesized ( $\beta$-CD)-SNPs show a high peak of absorbance between wavelengths of $400-460 \mathrm{~nm}$ and specifically at $453 \mathrm{~nm}$. The UV-Visible absorption spectrum of the ( $\beta-C D)-$ SNPs in solution is shown in Figure 4.

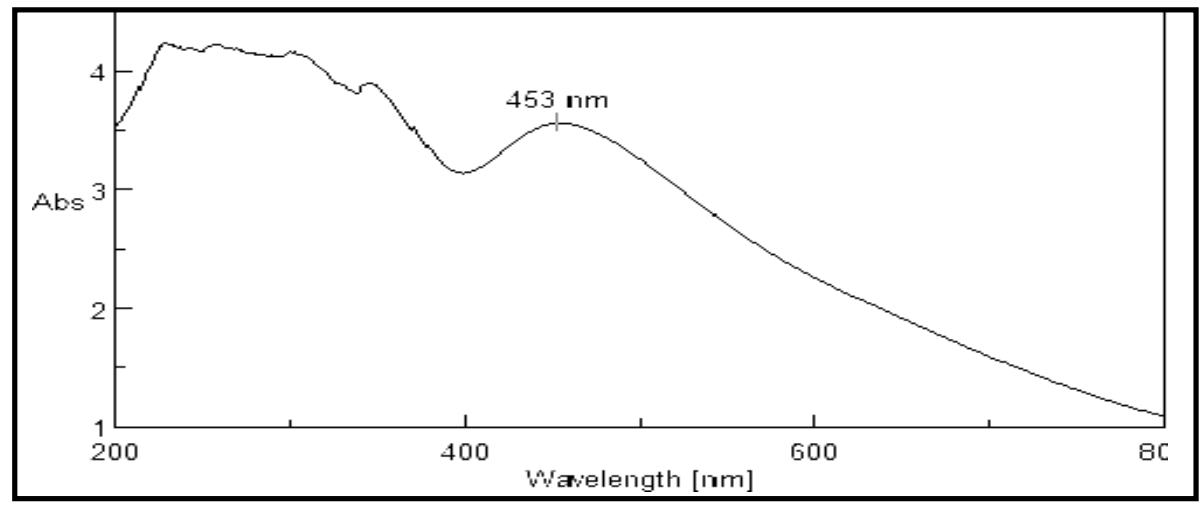

Figure 4. UV-Visible absorption spectrum of the $\beta$ - Cyclodextrin Silver Nanoparticles ( $\beta$ CD-SNPs).

3.2.2 Fourier Transform Infra-Red (FTIR) spectrum of $\beta$ CD-SNPs.

The FTIR spectrum for $\beta$-Cyclodextrin Silver Nanoparticles $(\beta C D-S N P)$ and its corresponding functional groups present is shown in Figure 5 and Table 1, respectively. 


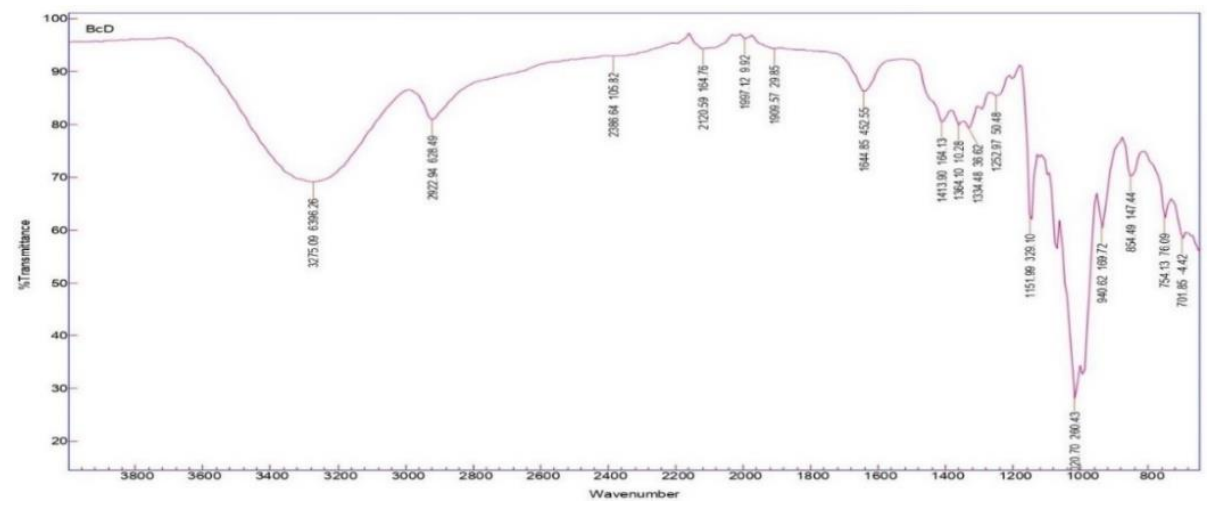

Figure 5. FTIR spectrum of ( $\beta$-CD)-SNPs in the range of $981.77 \mathrm{~cm}^{-1}-3317.56 \mathrm{~cm}^{-1}$. It shows that the solution of $\beta$ - cyclodextrin has the ability to perform dual functions of reduction and stabilization of silver nanoparticles.

Table 1. Shows functional group detected on the basis of FTIR for $\beta$-cyclodextrin silver nanoparticles $(\beta-C D)$ SNPs with its absorption range, bond, functional group.

\begin{tabular}{l|l|l|l}
$\begin{array}{l}\text { Sr } \\
\text { no }\end{array}$ & Absorption & Types of vibration & Class of compounds \\
\hline $\mathbf{1 .}$ & $\begin{array}{l}701336,746.102, \\
835.635,910.245\end{array}$ & C-Cl Stretch & Halo Compound \\
\hline $\mathbf{2 .}$ & $1014.699,1141.537$ & C-N Stretch & Amine \\
\hline $\mathbf{3 .}$ & 1234.800 & C-O Stretch & Aromatic ester \\
\hline $\mathbf{4 .}$ & $1334.332,1357.906$, & O-H Stretch & Alcohol \\
\hline $\mathbf{5 .}$ & 1398.942 & & \\
\hline $\mathbf{6 .}$ & $1902.561,1984.633$ & C-H Stretch & Nitro compound \\
\hline $\mathbf{7 .}$ & 2077.895 & C $\equiv$ C Stretch & Phenyl ring substitute \\
\hline $\mathbf{8 .}$ & 2275.612 & C=O & $\alpha, \beta$ unsaturated ketone \\
\hline $\mathbf{9 .}$ & $2730.735,3133.630$ & C-H Stretch & Ester \\
\hline
\end{tabular}

\subsubsection{Zeta potential ( $\beta$-CD)-SNPs.}

For zeta potential measurements confirmed that the zeta potential value was determined as $-9.37 \mathrm{mV}$, which confirms particles have a negative charge in water. This negative value indicates the stability of the nanoparticles, and the result is shown in Figure 6.

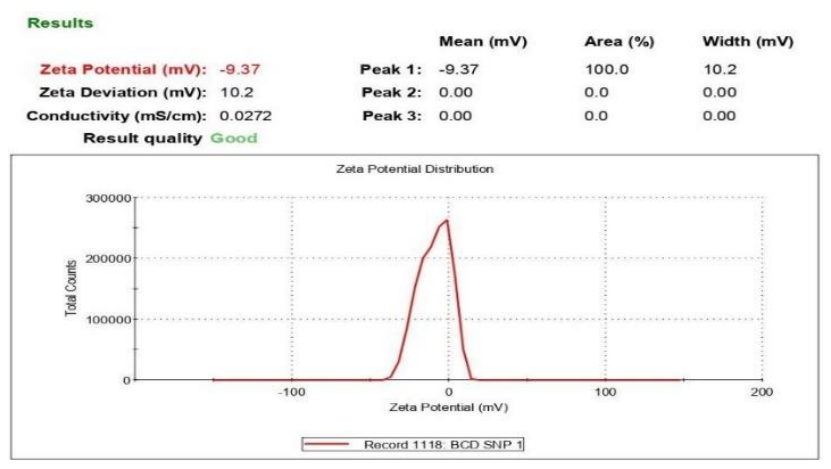

Figure 6. Shows the zeta potential of $\beta$-cyclodextrin silver nanoparticles ( $\beta$-CD)-SNPs.

3.2.4. Nanoparticle tracking analysis of ( $\beta-\mathrm{CD})$-SNPs.

The particle size of ( $\beta$-CD)-SNPs was found to be 130nm (Figure 8) and also confirms the uniform distribution of particles (Figure 7). 


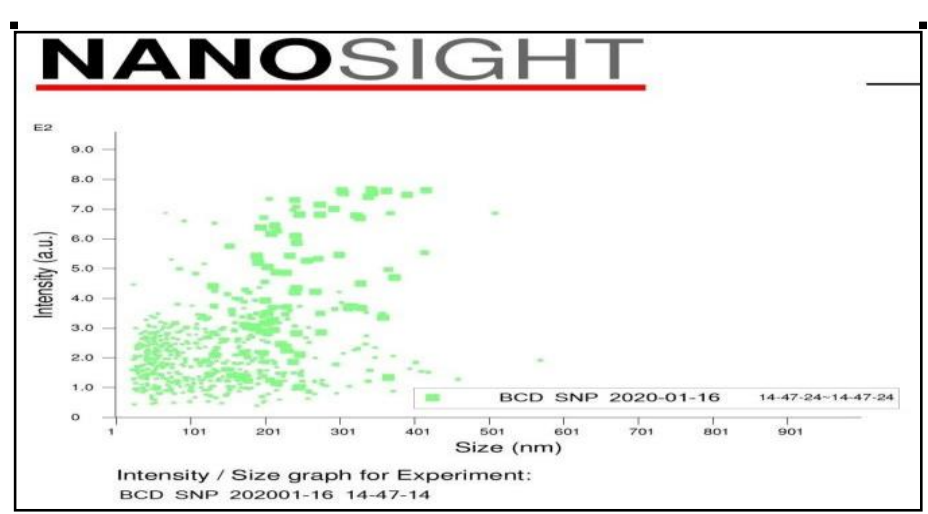

Figure 7. 3-D plot distribution of $\beta$-cyclodextrin silver nanoparticles ( $\beta$-CD)-SNPs characterized by Nanosight (LM 20). The size of nanoparticles was found to be less than $130 \mathrm{~nm}$ in size, where most of the nanoparticles are less than $400 \mathrm{~nm}$ in size with equal size distribution.

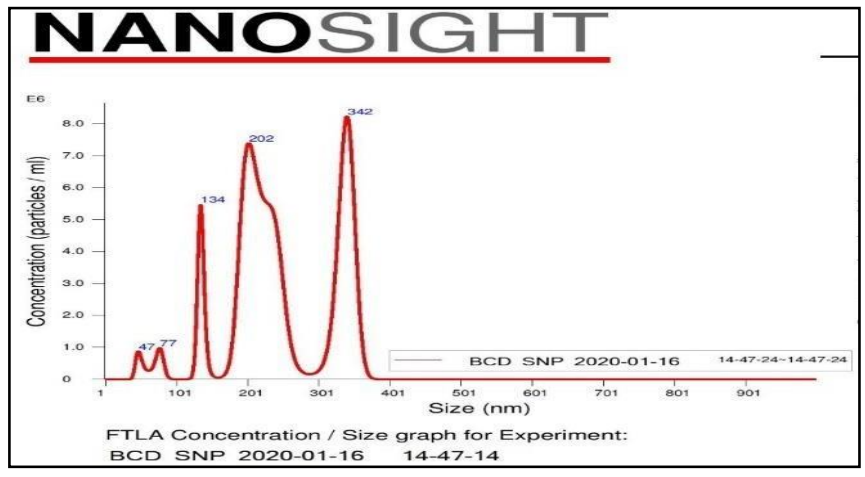

Figure 8. NTA (Nanosight-LM20) analysis of $\beta$-cyclodextrin silver nanoparticles ( $\beta$-CD)-SNPs size distribution histogram showing that the ( $\beta C D$-SNP) have a size between $47 \mathrm{~nm}$ to $342 \mathrm{~nm}$ with an average size of $130 \mathrm{~nm}$.

\subsection{Behavioral analysis of zebrafish.}

The fish behavior was evaluated visually after $0,3,6$, and $24 \mathrm{~h}$ and the following one month both in light as well as the dark period after exposure to silver nanoparticles. Four experimental conditions were considered in this study (Control tank, Tank \#1:30 $\mu 1 / \mathrm{L}$, Tank\#2:150 $\mu 1 / \mathrm{L}$, Tank\#3:300 $\mu \mathrm{l} / \mathrm{L})$. The $(\beta-\mathrm{CD})$-SNPs treated fishes showed dose-dependent toxicity under laboratory conditions. The control group appeared normal throughout the test period. In accordance with the use of NOEC (No observed effect concentration) concentrations, no mortality occurred in any treatment during the course of the exposure, and there were no alterations in zebrafish appearance, behavior, or movement patterns, for example, no visible lesions, uncoordinated swimming, or air-breathing. In the nanometal exposure containers, ingestion and excretion of nanometal particle aggregates were visually observed.

\subsubsection{Histopathology of zebrafish organs treated with ( $\beta-C D)-S N P s$.}

The individual gills, liver, and kidney from ( $\beta$-CD)-SNPs exposure as well as from the control group were analyzed separately for tissue metal concentration. Tissues were analyzed for total metal content via the classic histopathological process.

\subsubsection{Gill histopathology.}

Morphological responses of the gills to metal exposure were characterized by increased cellularity in the interlamellar space. Changes in gill filament width following silver 
nanoparticle exposure varied significantly. Soluble silver nanoparticle exposure both increased gill filament widths by approximately twofold over control values. Gills took from Tank \#1 (conc. $30 \mu \mathrm{l} / \mathrm{L}$ ) nanoparticle exposed fish were significantly wider than those from other $(\beta$ CD)-SNPs exposed tanks, highlighting the presence of a silver nanoparticle-specific effect (Figure 9). Entire gill arches were removed from zebrafish after 10 days and immediately frozen until analysis. The gills are the primary site of exposure for Ag- NP resulting from disruption of osmotic balance. The exposure to ( $\beta$-CD)-SNPs caused damage to the structure of the gills, such as sub-epithelial edema, hyperplasia, lamellar fusion, and telangiectasia. The scale barr: $\mathrm{A}=110 \mu \mathrm{m} ; \mathrm{B}=70 \mu \mathrm{m}$.

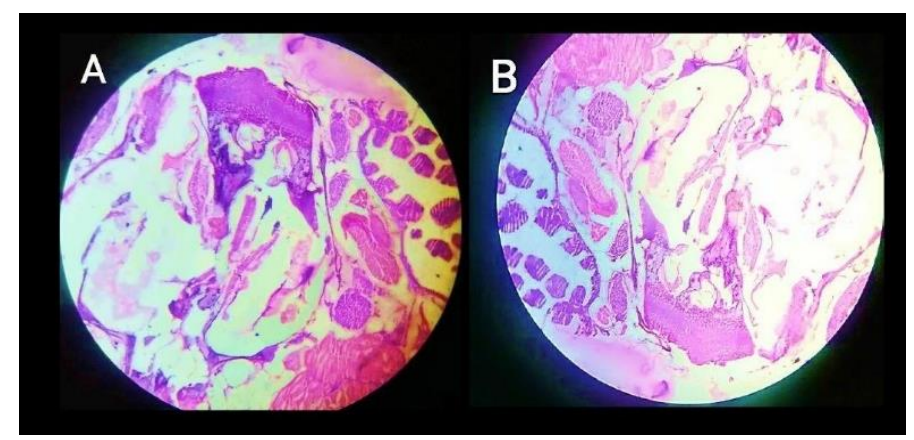

Figure 9. Gills morphology images of zebrafish treated with (A) Tank \#1 (conc. 30 $\mu 1 / \mathrm{L}$ ) and (B) Tank \#3 (conc. $300 \mu \mathrm{l} / \mathrm{L}$ ) exposure group respectively. This gills structure are recorded after 10 days of exposure

\subsubsection{Liver histopathology.}

Because the liver (made up of hepatic cells) is the primary organ for chemical detoxification, it is frequently a target for toxicity caused by engineered materials (here silver nanoparticles). In zebrafish, hepatotoxicity is accompanied by significant cell death due to necrosis and degenerative alterations, as well as a massive increase in nitric oxide levels, resulting in oxidative stress. That being said, the use of larger ( $\beta$-CD)-SNPs, $130 \mathrm{~nm}$ did not demonstrate a similar hepatotoxic effect, and total protein levels in the plasma were unaltered following acute exposure of ( $\beta$-CD)-SNPs, thereby confirming liver health (Figure 10). The Liver structure after 10 days of exposure $(\beta-C D)$-SNPs showed no cytological alterations in any fish groups.

\subsubsection{Kidney histopathology.}

The exposure of ( $\beta$-CD)-SNPs to zebrafish for examining bio localization demonstrated that Ag-NPs could disseminate throughout the body and Ag+ can accumulate in all major organs with the highest loads observed in the kidney (Figure 11). The fish did not die in the 10 days period due to these cellular changes, and the study was not followed up to determine if longer exposure, or chronic exposure would cause mortality. The kidney structure after 10 days of exposure ( $\beta$-CD)-SNPs showed major cytological alterations in: (A) Tank \#1 fish group; (B) Tank \#3 fish group. It can be clearly seen that $(\beta-C D)$-SNPs are more densely embedded in

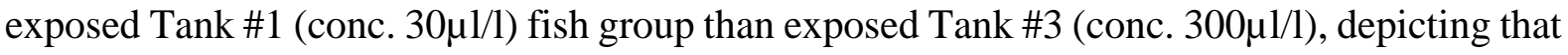
more SNPs are absorbed in less concentration rather than a larger concentration group, implying toxicity depends on concentration. 


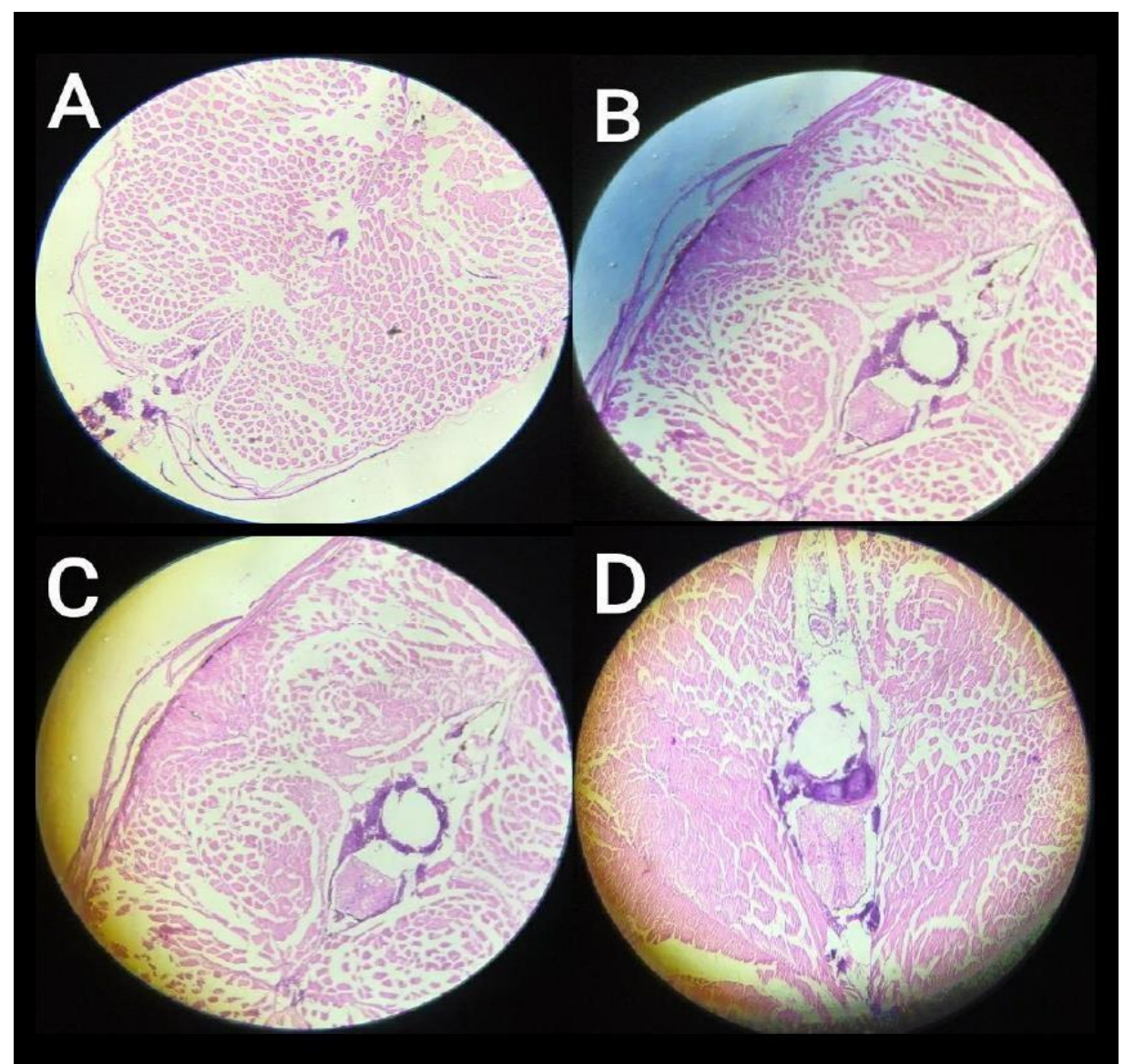

Figure 10. Liver morphology images of zebrafish treated with $\mathbf{A}$ (control tank), B (tank\#1 conc.30 $\mu 1 / \mathrm{L}), \mathbf{C}$ (tank\#2 conc. $150 \mu 1 / \mathrm{L}$ ) and $\mathbf{D}$ (tank\#3 conc.300 $\mu \mathrm{l} / \mathrm{L})$. The liver structure after 10 days of exposure to $\beta$ cyclodextrin silver nanoparticles $(\beta-\mathrm{CD})$-SNPs showed no cytological alterations in any fish groups.

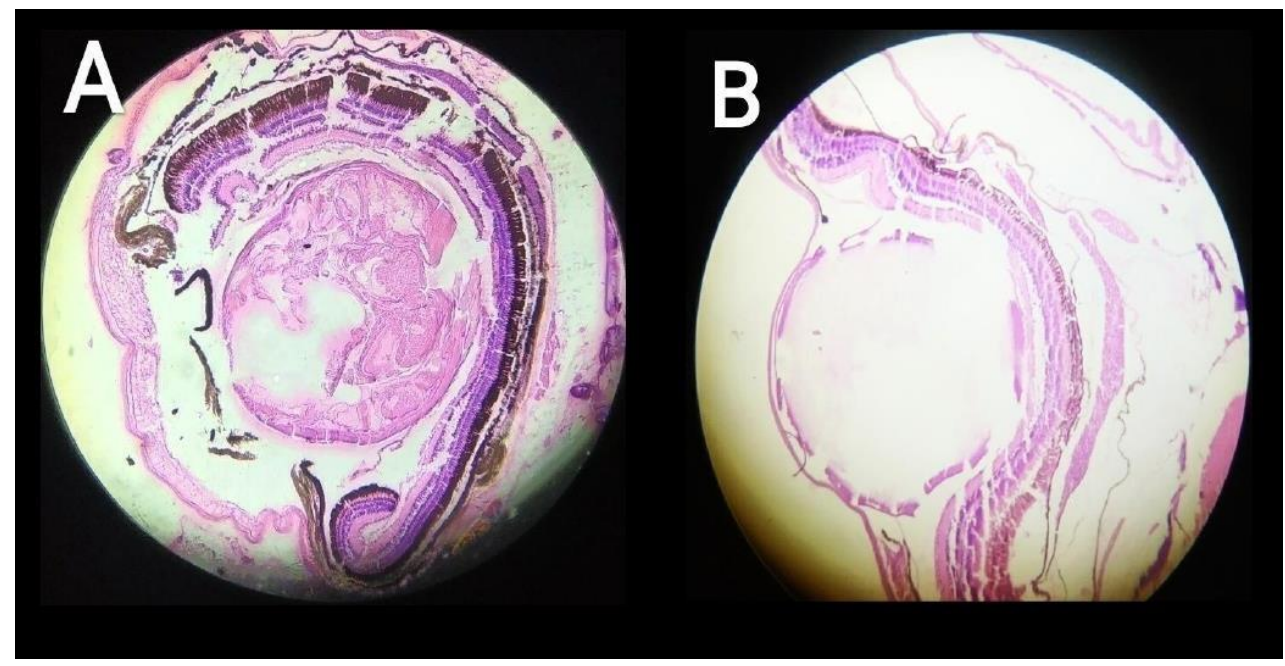

Figure 11. Kidney organ morphology images of zebrafish treated with (A) Tank \#1 (conc. 30 $\mu 1 / L)$ exposure

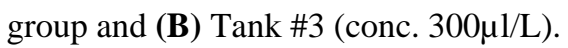

The prepared aqueous solution of $\beta$-Cyclodextrin $(\beta C D)$ was colorless, upon reaction with the silver salt, after adding few drops of $\mathrm{NaOH}$ solution from colorless to greyish-black color (Figure 6). This color change indicates the formation of Ag nanoparticles that are reduced due to the involvement of functional groups in the structure of $\beta$-cyclodextrin $(\beta C D)$. The color changes are possible because some of the $\mathrm{Ag}$ ions begin to be reduced due to heat and produce Ago stable atoms. 
The ( $\beta$-CD)-SNPs synthesized were analyzed using UV-Vis spectroscopy to determine the characteristics of the peak spectrum of the $\mathrm{Ag}$ nanoparticle wavelength prepared for $\mathrm{AgNO}_{3}$ concentration $(1 \mathrm{mM})$. For $1 \mathrm{mM}$, the absorption peak is centered around $400-460 \mathrm{~nm}$ for $(\beta-$ CD)-SNPs. This information shows that the Ag nanoparticles have formed in the solution, where the Ag+ has been reduced to Ag0 [8]. The solution's UV-Visible absorption spectrum of ( $\beta$-CD)-SNPs is $453 \mathrm{~nm}$ (Figure 4). The characteristics of silver nanoparticles normally appear at a wavelength interval of $400-600 \mathrm{~nm}$, and the work of [16] suggested that Ag-BSA nanoparticles showed maximum absorbance at $424 \mathrm{~nm}$. Earlier reports proved that silver nanoparticles are more lethal to cell-based in vitro systems than other metal nanoparticles screened. The effects of silver nanoparticles are frequently compared to the effects of silver ions, which are one of the most toxic ions for fish, disrupting osmoregulation due to the inhibition of gill $\mathrm{Na}+\mathrm{K}+-\mathrm{ATPase}$ [17]. The nanoparticles used in this study showed remarkable stability and uniform dispersion throughout the test period. No agglomeration and precipitation were observed. Nanoparticle stability is a major concern in nanotechnology and nanotoxicology. An unstable nanoparticle will precipitate to a metal clump which affects the test interpretations. In the case of water-insoluble particles, the carrier solvents may exert independent toxic effects in embryos. Hence it is ideal to use water as a carrier solvent to test nanoparticle toxicity. The use of water-insoluble nanoparticles could be less effective owing to phase separation.

The FTIR absorption spectra of ( $\beta$-CD)-SNPs were found to be in a range of 701.849 $\mathrm{cm}-1-3275.086 \mathrm{~cm}-1$. The three main bands could be observed in Figure 5. The broadband appearing at $1334.476 \mathrm{~cm}^{-1}$ is assigned for $\mathrm{O}-\mathrm{H}$ stretching vibration, indicating the presence of hydroxyl groups in the reducing agent. The strong, intense peaks at $1014.699 \mathrm{~cm}-1$ and $1141 \mathrm{~cm}-1$ corresponds to $\mathrm{C}-\mathrm{N}$ stretch vibrations as well as to the amide I band of proteins in $(\beta-C D)$-SNPs. Our result of the FTIR spectroscopic study confirmed that the $\beta$-cyclodextrin solution has the ability to perform dual functions of reduction and stabilization of silver nanoparticles [8]. The zeta potential of the synthesized ( $\beta$-CD)-SNPs was found to be $-9.37 \mathrm{mV}$ (Figure 6), in contrast [17] synthesized the AgNP having the zeta potential of $-26.8 \mathrm{mV}$. The zeta potential value could be positive or negative; the negative potential value shown by ( $\beta$ CD)-SNPs could be due to the possible capping of the organic component ( $\beta$-cyclodextrin). In contrast, the colloids are least stable at the isoelectric point, with zero zeta potential.

From Figure 7, it is observed that the nanoparticles obtained are polydispersed mixtures in the range $47 \mathrm{~nm}$ to $342 \mathrm{~nm}$, with an average size of $130 \mathrm{~nm}$ achieved through nanoparticles tracking analysis (NTA). The [17] synthesized spherical AgNP of $30.00 \pm 16.80 \mathrm{~nm}$ size. In addition, similar to our study, the [18] have synthesized silver nanoparticles of average size around $150 \mathrm{~nm}$. The sizes and shapes of metal nanoparticles are influenced by several factors, including $\mathrm{pH}$, precursor concentration, reductant concentration, time of incubation, temperature, and preparation method [2].

Any observed bioaccumulation of silver in the fish body may have been due to both ( $\beta$ CD)-SNPs and free silver ions. This could be part of the reason why previous studies have chosen to compare the toxicity effects of AgNPs and silver ions [18]. The experimental group of zebrafish exposed to the highest concentration $(300 \mu \mathrm{l} / \mathrm{L})$ had a lower toxicity degree than the group treated with NPs lower concentrations $(30 \mu \mathrm{l} / \mathrm{L})$. These results suggested that as high the concentration is, as high is the probability that the particles aggregate and little by little as their size increases, the exposed tissues' absorption capacity may reduce. Instead, the solution with the lower concentration of silver nanoparticles is mono-dispersed and more easily 
adsorbed by gills, liver, and kidney. For this reason, it is possible to affirm that the toxicity of silver nanoparticles is related to their size and degree of dispersion rather than their concentration [19].

The gill morphology images (Figure 9 ) show gill structure after 10 days to exposure ( $\beta$ CD)-SNPs. Exposure to silver nanoparticles caused damage to the structure of the gills, such as sub-epithelial edema, hyperplasia, lamellar fusion, and telangiectasia. Similarly, Rahmani et al. (2016) studied the titanium dioxide NP effect on $D$. rerio. The histopathology analysis of liver of fish groups from control tank, tank\#1 $(30 \mu \mathrm{l} / \mathrm{L})$, tank\#2 $(150 \mu \mathrm{l} / \mathrm{L})$ and tank\#3 $(300 \mu \mathrm{l} / \mathrm{L})$ was unaltered after 10 days following acute exposure of ( $\beta$-CD)-SNPs, thereby confirming liver healthy. Kidney organ morphology images (Figure 10) show kidney structure after 10 days of exposure ( $\beta$-CD)-SNPs. It depicts major cytological alterations in the Tank \#1 fish group and Tank \#3 fish group. It can be clearly seen that $(\beta-C D)-S N P s$ are more densely

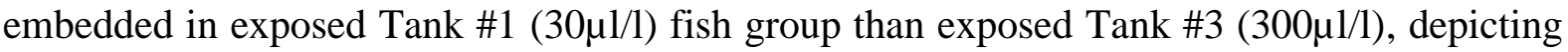
that more SNP is absorbed in less concentration rather than a larger concentration group, implying toxicity depends on concentration. Our results suggest that the toxicity of silver nanoparticles to aquatic species (zebrafish) depends on a concentration-dependent manner. Indicators of toxicity include the morphological section of gills, liver and kidney of zebrafish after a period of 10 days exposure to silver nanoparticles. Similarly, the [17] performed the study the effect of the AgNP concentrations on Danio rerio (zebrafish) tissues such as brain, muscle, gill, and liver tissues. Besides, [19] studies the toxic effect of AgNP on zebrafish at the gene expression and histopathology level.

The effect of different coatings (dextran, chitosan, polyethylene glycol, carboxy- silane, and silica) was studied in the toxicity elicited by superparamagnetic iron oxide nanoparticles (SPIONs) in the developing zebrafish (Danio rerio). The magnetic nanoparticle-based toxicity was evaluated on Embryo-Larvae stages of zebrafish [20]. In contrast, [16] studied the effect of silver nanoparticles synthesized using starch and bovine serum albumin (BSA) as capping agents to identify their distribution pattern in zebrafish embryos (Danio rerio). They found that concentration-dependent toxicity and stabilizing agents showed no significant defects in developing embryos. Our results obtained through the different applied methods showed that silver nanoparticles had caused tissue damage because they were absorbed in the body. It is conceivable that the nanoparticles could enter the cells through many routes, including diffusion or endocytosis through embryos' skin. This is supported by the presence of clumps of nanoparticles throughout the epidermis of the dissected sections [16] showed that nanoparticles were distributed in the brain, heart, yolk, and blood of embryos confirmed through electrondispersive $\mathrm{x}$-ray analysis (EDS).

In aquaculture, the studies of ( $\beta$-CD)-SNPs effects on zebrafish will assist in developing the nanoparticles-based drug that may be developed further to apply in future research. In vivo studies on the toxicokinetics of different engineered nanomaterials demonstrated their ability to be absorbed through many ways and reach through the bloodstream or lymphatic system, different organs and tissues, which can lead to adverse health effects.

\section{Conclusions}

The $\beta$-Cyclodextrin has been used successfully to reduce and capped silver salt to form ( $\beta$-CD)-SNPs successfully using $1 \mathrm{mM}$ silver nitrate (AgNO3) and $1 \% \beta$-cyclodextrin at $\mathrm{pH} 8$. The UV-Visible spectroscopy revealed a peak at characteristics wavelength at $453 \mathrm{~nm}$ specific for $(\beta-C D)-S N P s$. The Fourier Transformed Infra-Red spectrum of ( $\beta$-CD)-SNPs shows an 
absorbance band at suggesting their involvement in capping and reducing the synthesized ( $\beta$ CD)-SNPs. The zeta potential analysis confirmed that the synthesized ( $\beta$-CD)-SNPs have surface charges of $-9.37 \mathrm{mV}$ value indicated their stability. The size of $(\beta-C D)$-SNPs was found to be $130 \mathrm{~nm}$ using the nanoparticle tracking analysis. The $-9.37 \mathrm{mV}$ charged ( $\beta$-CD)-SNPs showed some effects on zebrafish organs studies using a microscope. The gills' structure after 10 days of exposure ( $\beta$-CD)-SNPs caused damage to the structure of the gills, such as subepithelial edema, hyperplasia, lamellar fusion, and telangiectasia. The histopathology analysis of the liver showed the liver healthy. The kidney organ morphology images exhibited kidney structure after 10 days of exposure ( $\beta$-CD)-SNPs are more densely embedded in exposed Tank $\# 1$ (conc. 30 $\mu 1 / 1$ ) fish group than exposed Tank \#3 (conc. 300 $\mu 1 / 1)$ depicting that more ( $\beta$-CD)SNPs are absorbed in less concentration rather than a larger concentration group, implying toxicity depends on concentration.

To sum up, the ( $\beta$-CD)-SNPs synthesized successfully and have some toxicity effects on some zebrafish organs that depend on the duration of exposure and concentration. Furthermore, it would be appropriate to make further examinations for the marine waters and establish new limits of the law to protect human health, animal species, and the environment after further investigations on nanoparticle toxicity.

\section{Funding}

No funding was available for this work.

\section{Acknowledgments}

The authors would like to thanks Rajiv Gandhi Biotechnology Centre, Rashtrasant Tukadoji Maharaj University, Maharashtra, India, for providing the lab facility for performing the experimentation. We would like to thank Narsamma Hirayya Arts Commerce \& Science College, 444605 Amravati, Maharashtra, India, for FTIR analysis and Department of Biotechnology, Sant Gadge Baba Amravati University Tapovan, Amravati 444602 Maharashtra, India, for zeta potential analysis. Also, to the Star Histopathology Lab, Plot no. 124 Old Subhedar Layout, Sharda Chowk, 440024 Nagpur, Maharashtra, India, for the histopathology of fish samples.

\section{Conflicts of Interest}

The authors declare that they have no conflict of interest.

\section{References}

1. Jeevanandam, J.; Barhoum, A.; Chan, Y.S.; Dufresne, A.; Danquah, M.K. Review on nanoparticles and nanostructured materials: history, sources, toxicity and regulations. Beilstein J. Nanotechnol. 2018, 9, 10501074, https://doi.org/10.3762/bjnano.9.98.

2. Lade BD and Shanware AS. Phytonanofabrication: Methodology and factor affecting synthesis of silver nanoparticles. Intechopen Smart Nanosystems for Biomedicine, Optoelectronics and Catalysis, Tatyana Shabatina and Vladimir Bochenkov. IntechOpen 2020, 1-17, https://doi.org/10.5772/intechopen.90918.

3. Lade, B.D.; Patil, A.S. Silver nano fabrication using leaf disc of Passiflora foetida Linn. Appl Nanosci. 2017, 7, 181-192, https://doi.org/10.1007/s13204-017-0558-y.

4. Krasniewska, G. S.; Gniewosz, M. Biopolymers-Based Materials Containing Silver Nanoparticles as Active Packaging for Food Applications-A Review. Int. J. Mol. Sci. 2020, 21, 698, https://doi.org/10.3390/ijms21030698. 
5. Yurii, K.; Gun, k. Nanoparticles in Bioimaging, Nanomaterials 2016, 6, 105, https://doi.org/10.3390/nano6060105.

6. Mody, V.V.; Siwale, R.; Mody, H.R. Introduction to metallic nanoparticles. Journal of Pharmacy and Bioallied Sciences. 2010, 2 (4), 282-289, https://doi.org/10.4103/0975-7406.72127.

7. Alexander, J. W. History of the medical use of silver. Surg. Infect. (Larchmt) 2009, 10, 289-292, https://doi.org/10.1089/sur.2008.9941.

8. Celebioglu, A.; Uyar, T. Electrohydrodynamic encapsulation of eugenol-cyclodextrin complexes in pullulan nanofibers, Food Hydrocolloids 2021, 111, 106264, https://doi.org/10.1021/acs.jafc.7b04312.

9. Loftsson, T.; Masson, M.; Brewster, M.E. Selfassociation of cyclodextrins and cyclodextrin complexes. J. Pharm. Sci. 2001, 93, 1091-1099, https://doi.org/10.1016/s0378-5173(01)00761-x.

10. Loftsson, T.; Fridrikdottir, H.; Sigurdardottir, A.M.; Ueda, H. The effect of water-soluble polymers on drugcyclodextrin complexation. Int. J. Pharm. 1994, 110, 169-177, https://doi.org/10.1016/0378-5173(94)901945.

11. Sanders, J.L.; Watral, V.; Kent, M.L. Microsporidiosis in Zebrafish Research Facilities. ILAR Journal 2012, 53, 106-113, https://doi.org/10.1093/ilar.53.2.106.

12. Meyers JR (2018). Zebrafish: Development of a Vertebrate Model Organism. Current protocol 2018, 16, https://doi.org/10.1002/cpet.19.

13. D'Costa, A.; Shepherd, I. Zebrafish Development and Genetics: Introducing Undergraduates to Developmental Biology and Genetics in a Large Introductory Laboratory Class. 2009, 412, 71-75, https://doi.org/10.1089/zeb.2008.0562.

14. Pecoraro, R.; Marino, F.; Salvaggio, A.; Capparucci, F.; Caro, G.D.; Iaria, C.; Salvo, A.; Rotondo, A.; Tibullo, D.; Guerriero, G.; Scalisi, E.M.; Zimbone, M.; Impellizzeri, G.; Brundo M.V.Evaluation of Chronic Nanosilver Toxicity to Adult Zebrafish. Aquatic physiology 2017, 1-9, https://doi.org/10.3389/fphys.2017.01011.

15. Rahmani, R.; Mansouri, B.; Azadi, N.A.; Davari, B.; Johari, A.S.; Maleki, A.; Pordel, M.A. Histopathological alterations in the gill of zebrafish (Danio rerio) exposed to $\mathrm{Cr}$ and $\mathrm{Ba}$ doped $\mathrm{TiO}_{2}$ nanoparticles. AACL Bioflux, 2016, 9:4, Microsoft Word - 2016.889-900.doc (researchgate.net).

16. Asharani, P.V.; Lian, W. Y.; Gong, Z.; Valiyaveettil, S. Toxicity of silver nanoparticles in zebrafish models. Nanotechnology 2008, 19, 255102, https://doi.org/10.1088/0957-4484/19/25/255102.

17. Morgan, T. P.; Wood, C. M. A relationship between gill silver accumulation and acute silver toxicity in the freshwater rainbow trout: support for the acute silver biotic ligand model, Environmental Toxicology and Chemistry 2004, 23, 1261-1267, https://doi.org/10.1897/03-181

18. Marinho, C.S.; Matias, M.V.F.; Toledo, E.K.M.; Smaniotto, S.; Ximenes-da-Silva, A.; Tonholo, J.; Santos, E.L.; Machado, S.S.; Zanta, C.L.P.S. Toxicity of silver nanoparticles on different tissues in adult Danio rerio. Fish Physiol Biochem. 2020, 1-1, https://doi.org/10.1007/s10695-020-00909-2.

19. Umoren, S.A.; Obot, I.B.; Gasem, Z.M. Green Synthesis and Characterization of Silver Nanoparticles Using Red Apple (Malus domestica) Fruit Extract at Room Temperature. J. Mater. Environ. Sci. 2014, 5 (3), $907-$ 914, CiteSeerX — Green Synthesis and Characterization of Silver Nanoparticles Using Red Apple (Malus domestica) Fruit Extract at Room Temperature (psu.edu).

20. Griffitt, R.J.; Luo, J.; Gao, J.; Bonzongo, J. C.; Barber, D.S. Effects of particle composition and species on toxicity of metallic nanomaterials in aquatic organisms. Environ. Toxicol. Chem. 2008, 27, 1972-1978, https://doi.org/10.1897/08-002.1. 